\title{
Diagnosis of nerve root avulsion injuries in adults with traumatic brachial plexopathies: MRI compared with CT myelography
}

\begin{abstract}
Authors:
Ewoudt van der Linde

Vanesha Naidu ${ }^{1}$

Ayesha Mitha ${ }^{2}$

Antoine Rocher ${ }^{3}$

Affiliations:

${ }^{1}$ Department of Radiology,

University of KwaZulu-Natal,

South Africa
\end{abstract}

${ }^{2}$ Department of Radiology Inkosi Albert Luthuli Central Hospital, South Africa

${ }^{3}$ Department of Orthopedics, Inkosi Albert Luthuli Central Hospital, South Africa

\section{Correspondence to:}

Ewoudt van der Linde

Email:

ewoudt@eventdoctors.co.za

Postal address:

PO Box 171, Hyper by the

sea, Durban 4053,

South Africa

Dates:

Received: 23 Jan. 2015

Accepted: 20 Jun. 2015

Published: 15 Oct. 2015

How to cite this article: Van der Linde, E, Naidu, $V$, Mitha, A, Rocher, A.

Diagnosis of nerve root avulsion injuries in adults with traumatic brachial plexopathies: MRI compared with CT myelography. S Afr J Rad. 2015;19(1); Art. \#779,

9 pages. http://dx.doi. org/10.4102/sajr.v19i1.779

\section{Copyright:}

(C) 2015. The Authors.

Licensee: AOSIS

OpenJournals. This work is

licensed under the Creative Commons Attribution

License.

\section{Read online:}

Background: Computed tomography myelography (CTM) has been broadly adopted as the 'gold standard' imaging technique in the diagnosis of nerve root avulsion injuries in traumatic brachial plexopathies. CTM has the distinct advantage of better spatial resolution than magnetic resonance imaging (MRI); however, this technique is invasive and can result in significant patient discomfort. MRI, therefore, seems relatively more advantageous as it is less invasive (requires no lumbar puncture), lacks radiation exposure, has no adverse reactions related to intrathecal contrast agents and confers excellent soft-tissue contrast.

Objectives: To compare the sensitivity and specificity of MRI with CTM in the diagnosis of preganglionic nerve root avulsion injuries in adults with traumatic brachial plexopathies at the Inkosi Albert Luthuli Central Hospital.

Method: A retrospective comparative analysis was performed on 16 adult patients with traumatic preganglionic brachial plexopathies who underwent both MRI and CTM at Inkosi Albert Luthuli Central Hospital. Radiologists experienced in both CTM and MRI interpreted the data and a comparison was made using CTM as the gold standard.

Results: The sensitivity and specificity for MRI detecting preganglionic nerve root avulsion injuries and pseudomeningoceles was $82 \%$ and $100 \%$ respectively. The interobserver agreement between CTM and MRI for the detection of preganglionic nerve root avulsion injuries was $81.25 \%(\mathrm{Kappa}=0.77)$ and $87.5 \%(\mathrm{Kappa}=0.84)$ for the detection of pseudomeningoceles.

Conclusion: MRI was as sensitive as CTM at detecting preganglionic nerve root avulsion injuries and pseudomeningoceles of spinal nerve roots $\mathrm{C} 7-\mathrm{T} 1$ of the brachial plexus. Some mild discrepancies existed at the $\mathrm{C} 5$ and $\mathrm{C} 6$ nerve root levels. Owing to the invasiveness of the procedure and resultant patient discomfort, CTM should be reserved for complicated cases or for patients with contraindications to MRI.

\section{Introduction}

The brachial plexus is a complex network of nerves that originate in the neck and is formed from the ventral rami of $\mathrm{C} 5$ to $\mathrm{T} 1$. It gives rise to numerous nerves that control motor and sensory function of the upper limbs.

Motorcycle accidents are the most common cause of all brachial plexus injuries in adults resulting from forced traction applied to the nerves (C5-T1) of the brachial plexus. The head and shoulders are forced apart and cause either nerve root avulsion injuries or stretch/rupture injuries. Preganglionic injuries are located proximal to the dorsal root ganglion whilst postganglionic injuries are located distal to the dorsal root ganglion. ${ }^{1}$

Differentiating between preganglionic and postganglionic brachial plexopathies is a crucial distinction as it determines patient management. Nerve transfers are used in the management of preganglionic injuries whilst postganglionic injuries are repaired with nerve grafting or managed conservatively. ${ }^{2}$ Most surgeons (94\%) operating on brachial plexopathies would prefer either computed tomography myelography (CTM) or magnetic resonance imaging (MRI) as their investigations of choice whilst $41 \%$ would prefer using both CTM and MRI. ${ }^{3}$ CTM is the imaging modality of choice in the diagnosis of preganglionic brachial plexopathies, with MRI as an additional investigation, whilst MRI alone is preferred in postganglionic injuries for visualising damaged peripheral nerves. , $^{2,5}$

Nerve root avulsion injuries are further classified as either partial or complete. Partial avulsion refers to avulsion of either the dorsal or ventral nerve root, whereas complete avulsion is the avulsion of both ventral and dorsal nerve roots. ${ }^{4}$ 
As a result of the direct visualisation of ventral and dorsal nerve roots, traumatic pseudomeningoceles are no longer regarded as the only incontrovertible evidence for nerve root avulsion injuries. ${ }^{6}$ Nerve roots can be identified on multi-axial CTM or MRI slices and should be compared with the contralateral intact nerve roots to avoid false-positive findings.

Although CTM is the gold standard for detecting nerve root avulsion injuries in traumatic brachial plexopathies, debate still exists whether it should not be replaced by MRI (because of all the advantages of MRI over CTM) and/or whether CTM should be reserved for patients with contraindications to MRI

\section{Method}

Formal ethics approval was obtained from the Biomedical Research Ethics Committee (BREC), College of Health Sciences, University of KwaZulu-Natal.

The study was conducted at Inkosi Albert Luthuli Central Hospital (IALCH), Durban. Data was collected retrospectively on all adult patients 18 years or older with traumatic preganglionic brachial plexopathies who underwent both cervical MRI and CTM from May 2012 to June 2014.

Jopamiron 300 (Iopamidol) was the contrast media used in all patients undergoing CTM. Under fluoroscopic guidance and aseptic conditions, contrast was introduced into the intrathecal space via a lumbar puncture. CTM of the cervical spine was performed approximately 45 minutes to 90 minutes after intrathecal injection using a Siemens Somatom Definition AS 128-slice computed tomography (CT) scanner with the following settings: Reconstructions $1 \mathrm{~mm} \times 1 \mathrm{~mm}$, Window - Inner ear, Kernel B70 (Very Sharp). Multiplanar reconstructed CT images of the cervical spine from C2-T3 were obtained.

MRI of the cervical spine was performed using a Siemens MAGNETOM Symphony 1.5T eco scanner. All images were obtained using a spinal cord coil, $256 \times 256$ matrix, and a 260 $\mathrm{mm}$ field of view (FOV). $\mathrm{T}_{2}$-weighted images ( $\mathrm{TR}=1500$, $\mathrm{TE}=126)$ with $1 \mathrm{~mm}$ slice thickness were obtained in three orthogonal planes.

Radiologists experienced in both CTM and MRI interpretation evaluated the images and retrospectively analysed the data. Image criteria for the diagnosis of nerve root avulsions were based on the absence of either one (partial avulsion) or both (complete avulsion) nerve roots. When both ventral and dorsal nerve roots were visualised, the nerve roots were documented as being intact. The evidence of pseudomeningoceles was also documented at the various levels it occurred. The analysis of each avulsed nerve root was compared to the contralateral normal/intact side in both CTM and MRI.
Patient demographics, injury lateralisation, cause of injury, nerve conduction study results, clinical examination, associated injuries and management were retrospectively analysed.

CTM and MRI findings were compared using CTM findings as the 'gold standard' (standard of reference). Sensitivity, specificity, as well as positive and negative predictive values were calculated and documented accordingly. The Kappa statistic (K-value) was used to evaluate interobserver agreement. $^{7}$

\section{Results}

Within a 2-year period, 16 patients were included in the study. Of these, 15 were male and only one female. The mean age was 30 years (youngest 19 years, oldest 43 years). All imaging was considered to be of diagnostic quality except for one CTM study that was interpreted as being of 'poor quality'. This was owing to the fact that contrast did not reach the cervical spine post fluoroscopic infiltration and was therefore not visualised on the CTM images. On average, CTM and MRI were done 98 days following sustained injury (lower limit $=40$ days; upper limit $=208$ days). CTM was the modality of first choice in 12 occasions. MRI was done before CTM in the remaining four occasions.

There was a total of seven (44\%) brachial plexus injuries on the left and nine (56\%) injuries on the right. The major causes of traumatic brachial plexopathies were motor vehicle accidents (69\%), pedestrian vehicle accidents (19\%), motorcycle accidents (6\%) and blunt trauma (6\%). The majority of patients had poly-trauma presenting with multiple upper and/or lower limb injuries.

Clinical presentation included pain, loss of sensation, motor function deficits, wasting of the muscles around the shoulder joint and scapular winging. Horner's syndrome was documented in two patients (13\%). Nerve conduction studies were performed on all patients; however, the majority of the results showed mixed sensory and motor deficits involving the $\mathrm{C} 5-\mathrm{T} 1$ nerve roots which were inconclusive.

In comparison to CTM, the sensitivity for MRI in the detection of preganglionic nerve root avulsion injuries and pseudomeningoceles was $82 \%$, the specificity $100 \%$, the positive predictive value (PPV) $100 \%$ and the negative predictive value (NPV) $71 \%$ (Figure 1). MRI had the same results as CTM for the detection of preganglionic nerve root avulsion injuries and pseudomeningoceles involving nerve roots C7-T1; however, CTM detected some pseudomeningoceles and preganglionic nerve root avulsion injuries at nerve roots C5/C6 of one patient, which was not detected on MRI. An epidural meningocele (EM) was detected in both CTM and MRI in one patient (Figures 2 and 3).

The interobserver agreement was measured using the Kappa statistic (K-value). The agreement between CTM and 


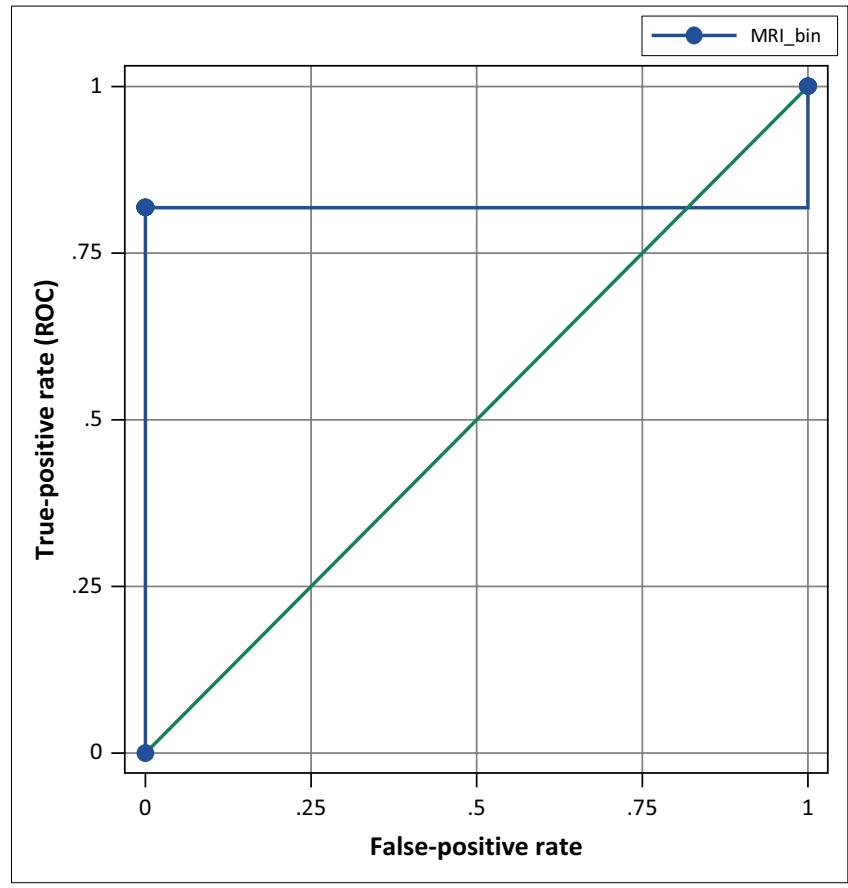

MRI, magnetic resonance imaging

FIGURE 1: Receiver operating characteristic (ROC) curve showing relationships between the sensitivity and specificity for magnetic resonance imaging compared with computed tomography myelography.

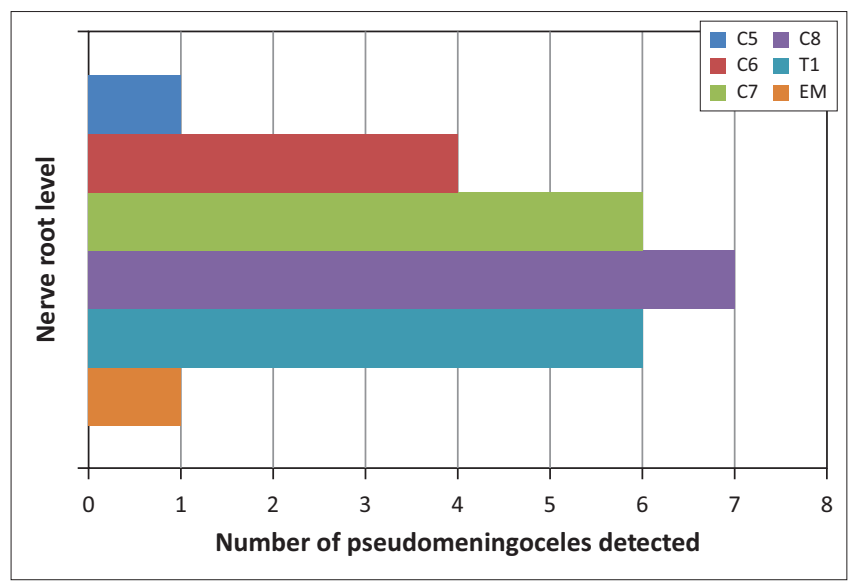

EM, epidural meningocele

FIGURE 2: Pseudomeningoceles and nerve root avulsion injuries detected by computed tomography myelography at the various nerve roots of the brachial plexus.

MRI for the detection of nerve root avulsion injuries was $81.25 \%(\mathrm{~K}=0.77)$ and $87.5 \%(\mathrm{~K}=0.84)$ for the detection of pseudomeningoceles.

All patients with confirmed preganglionic nerve root avulsion injuries were managed surgically with intercostal nerve transfers.

\section{Discussion}

Accurate assessment of the preganglionic nerve roots is imperative in the diagnosis of traumatic brachial plexopathies as it impacts on surgical approach and patient prognosis. Clinical examination and neurophysiological testing are

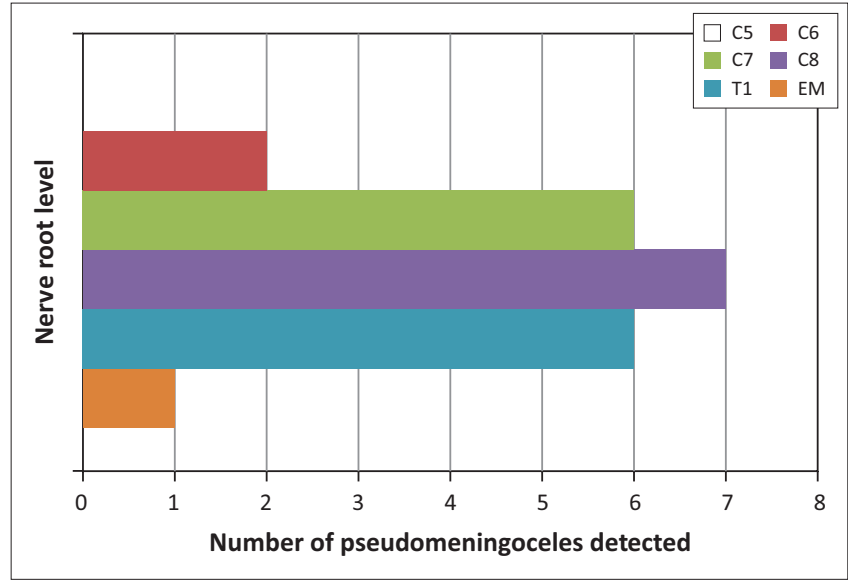

EM, epidural meningocele

FIGURE 3: Pseudomeningoceles and nerve root avulsion injuries detected by magnetic resonance imaging at the various nerve roots of the brachial plexus.

useful adjuncts that add great value to the diagnosis of these injuries.

The clinical assessment of the patient can guide radiological evaluation. Limthongthang et al. ${ }^{8}$ reported signs and symptoms that suggest preganglionic injuries, namely Horner syndrome (T1), winged scapula (C5-C7), atrophy of parascapular muscles (C5), cervical paraspinal muscle weakness and loss of posterior neck sensation, hemidiaphragm paralysis (C3-C5), pseudomeningocele on myelogram, and intact sensory nerve action potentials in the area of sensory deficit.

Neurophysiological testing plays an important role in the diagnosis of traumatic brachial plexopathies. ${ }^{9}$ Nerve conduction studies test for sensory and motor nerve functions. Motor nerve testing for detecting more distal nerve injuries is less helpful with traumatic brachial plexopathies when the muscles become completely denervated; however, conduction blocks may still be present in incomplete injuries. Sensory testing is one of the most important components of nerve conduction studies for traumatic brachial plexopathies. Sensory testing can assist in localising the site and severity of the plexus injury and is essential in differentiating preganglionic or postganglionic root injury. The cell body of the sensory nerve (dorsal root ganglion) resides outside the spinal cord but within the neural foramen. Nerve root avulsions that occur proximal to the foramen typically spare the dorsal nerve root ganglion, with sensory amplitudes remaining normal in preganglionic avulsion injuries. A reduction or unobtainable sensory response will be noted in high intraforaminal or extraforaminal injuries that involve the dorsal nerve root ganglia or peripheral nerve. Nerve traction injuries can cause segmental injuries resulting in mixed preganglionic and postganglionic components. ${ }^{9}$

However, clinical assessment and neurophysiological testing in isolation are not reliable enough and should always be reconciled with radiological imaging for a more accurate and 


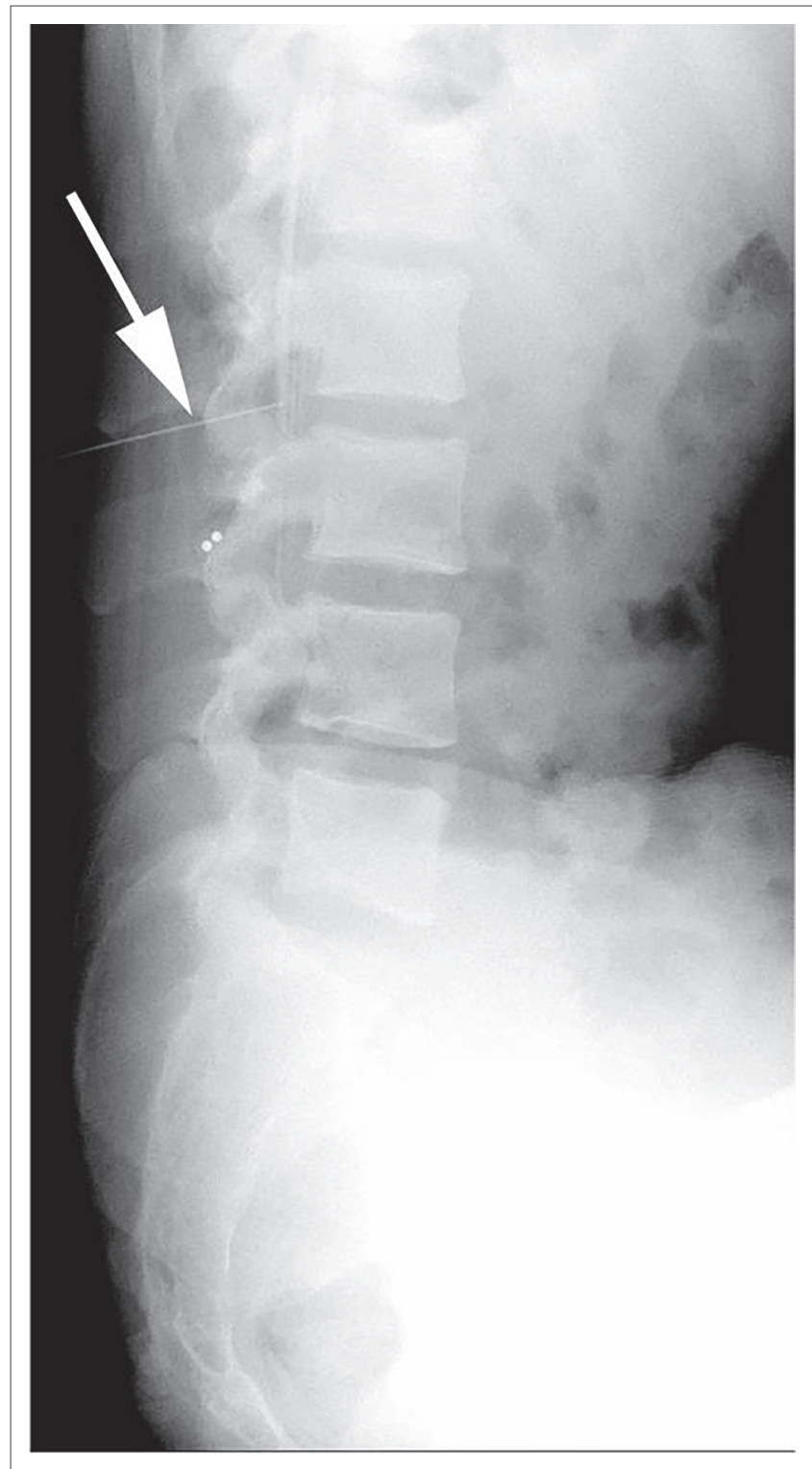

FIGURE 4: Lateral lumbar radiograph demonstrating the technique used for injecting contrast into the spinal canal under the guidance of fluoroscopy (L2/L3 level) using a spinal needle (arrow) for all patients undergoing computed tomography myelography.

holistic diagnosis. ${ }^{4} \mathrm{CTM}$ and MRI scans are undertaken to help the surgeon determine whether the brachial plexus injury is preganglionic, postganglionic or a combination of these.

CTM begins with an invasive procedure (lumbar puncture) whereby patients have contrast injected (under sterile technique) into the lumber spinal canal under fluoroscopic guidance (Figure 4). The patients are then transferred to the $\mathrm{CT}$ unit lying in a prone position with the head reclined to prevent contrast entering the intracranial cerebrospinal fluid (CSF) spaces. If the procedure is not performed adequately and timeously, contrast will not fill the correct CSF spaces, resulting in the $\mathrm{CT}$ being done with the contrast in the incorrect position (Figure 5). This reduces CTM sensitivity significantly and potentially exposes the patient to a higher radiation dose should the $C T$ examination need to be repeated. Further postprocedural complications such as headache, nausea and vomiting are also common. The advantages and disadvantages of CTM are presented in Table 1.

MRI is non-invasive and does not expose the patient to radiation. It offers good contrast resolution and clearly demonstrates the preganglionic nerve roots (Figure 6). The distal brachial plexus can also be visualised, which provides the surgeon with more information regarding the brachial plexopathy. MRI allows for better characterisation of the injury, differentiating preganglionic, postganglionic or combined lesions. MRI, therefore, confers a superior diagnosis that is more comprehensive than CTM. ${ }^{6}$ The advantages and disadvantages of MRI are presented in Table 2.

Images comparing MRI with CTM are shown in Figure 7. Pseudomeningoceles and preganglionic nerve root avulsion injuries are clearly visualised on both imaging modalities. Some discrepancies were noted, however, in a patient where CTM demonstrated C5/C6 nerve root avulsion injuries and pseudomeningoceles, which could not be visualised on the MRI study (Figure 8). This was thought to be as a result of technical factors.

Following a brachial plexus injury, any of the following or combinations may occur: avulsion, rupture or stretch injuries. Certain patterns are more prevalent:

- Supraclavicular regions are more affected than retroclavicular or infraclavicular regions. ${ }^{1}$

- Roots and trunks of the brachial plexus are much more affected than divisions, cords or terminal branches. ${ }^{1}$

- Approximately $70 \%-75 \%$ of injuries are found to be supraclavicular whilst $75 \%$ of these injuries involve an injury to the entire brachial plexus (C5-T1). ${ }^{1}$ In addition:

- $20 \%-25 \%$ of injuries involve injury to the nerve roots of C5-C7 and $2 \%-35 \%$ of injuries have isolated supraclavicular patterns to C8 and T1. ${ }^{1}$

- Panplexal injuries usually involve a C7-T1 nerve root avulsion together with a C5-C6 rupture. ${ }^{1}$

- The remaining $25 \%$ of brachial plexus injuries are infraclavicular. ${ }^{1}$

Although still controversial, Gasparotti et al. ${ }^{6}$ state that MRI and MRI myelography may offer many advantages over CTM and should be used as the primary investigation of choice when it comes to brachial plexopathies, whilst CTM should be reserved for patients with contraindications to MRI. Limthongthang et al. ${ }^{8}$ also reported a sensitivity of $92.9 \%$ and specificity of $81.3 \%$ for the detection of nerve root avulsion injuries using overlapping coronal-oblique MRI slices. Moreover, MRI was capable of producing useful images of the entire brachial plexus.

Yamazaki et al. ${ }^{10}$ demonstrated that MRI was superior to conventional and CT myelography for the visualisation of small meningoceles which did not fill with contrast medium in the presence of dural scars. Yamazaki et al. also showed that with CTM it was difficult to determine the exact level of the nerve root with axial imaging as 

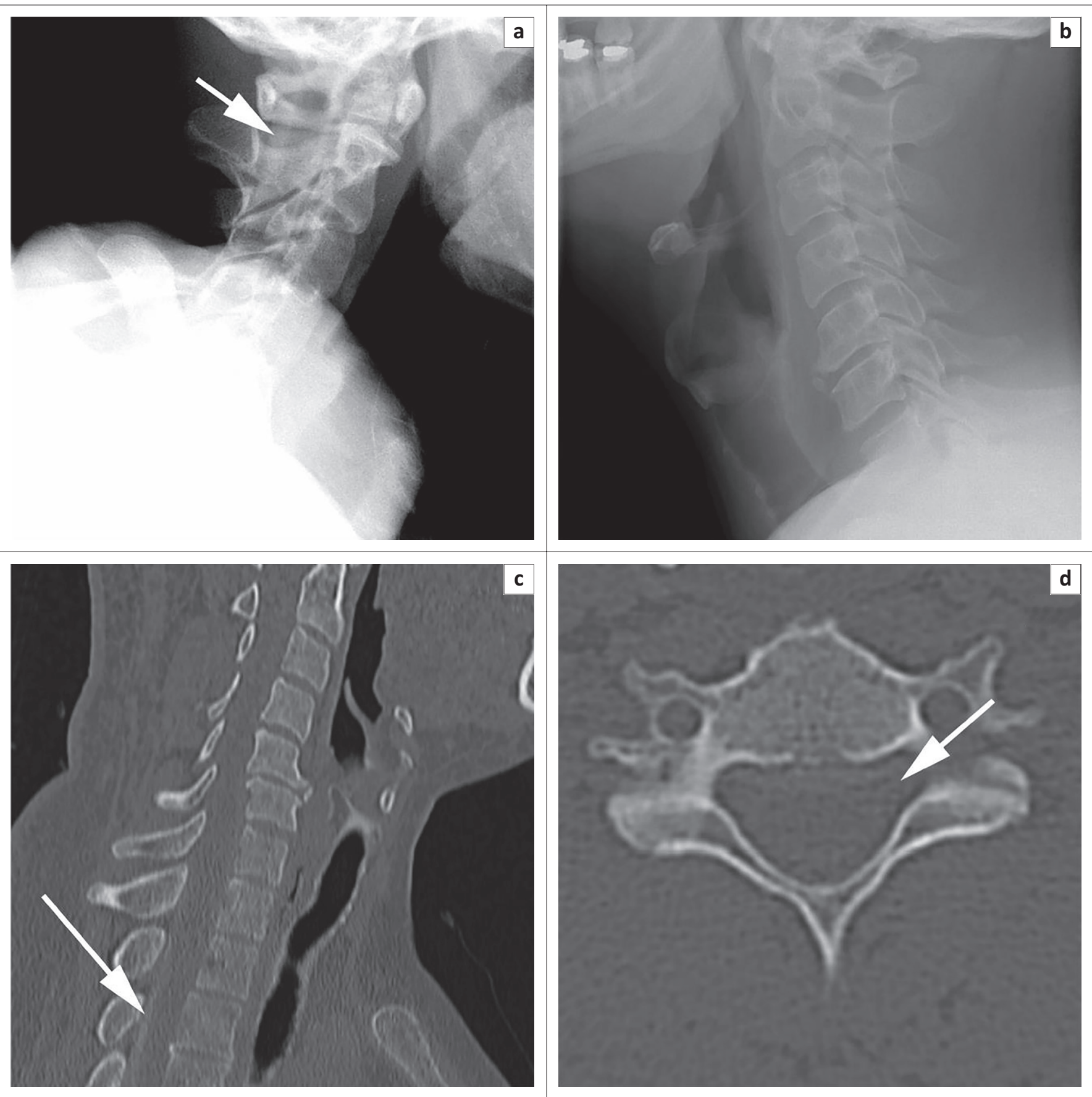

FIGURE 5: (a) Lateral radiograph of the cervical spine demonstrating contrast in the correct location (arrow) of the cervical spinal canal (post lumbar puncture) before undergoing the computed tomography (CT) scan. (b) Lateral radiograph of the cervical spine demonstrating no contrast visualised within the cervical spinal canal (post lumbar puncture), thus making it difficult for the radiologist to visualise the preganglionic nerve roots on axial CT scan images (arrow in d). (c) Lateral radiograph of the cervical-thoracic junction demonstrating the incorrect location of contrast that was noted within the upper thoracic spinal canal (arrow). This was as a result of technical inadequacy.

TABLE 1: Advantages and disadvantages of CTM.

\begin{tabular}{ll}
\hline Advantages of CTM over MRI & Disadvantages of CTM \\
\hline It can be used in claustrophobic patients & It is an invasive diagnostic procedure \\
It can reveal partial nerve root avulsions & It has high radiation exposure \\
There are no cerebrospinal fluid flow artefacts & Risk of adverse reaction \\
It has excellent visualisation of bone structures & Intraprocedural complications (epidural/subdural hematomas) \\
High-resolution multiplanar reconstructions can be used & Postprocedural complications (headache, seizures, nausea and vomiting) \\
- & Radiation beam hardening artefacts ${ }^{6}$ \\
\hline
\end{tabular}

CTM, computed tomography myelography; MRI, magnetic resonance imaging. 

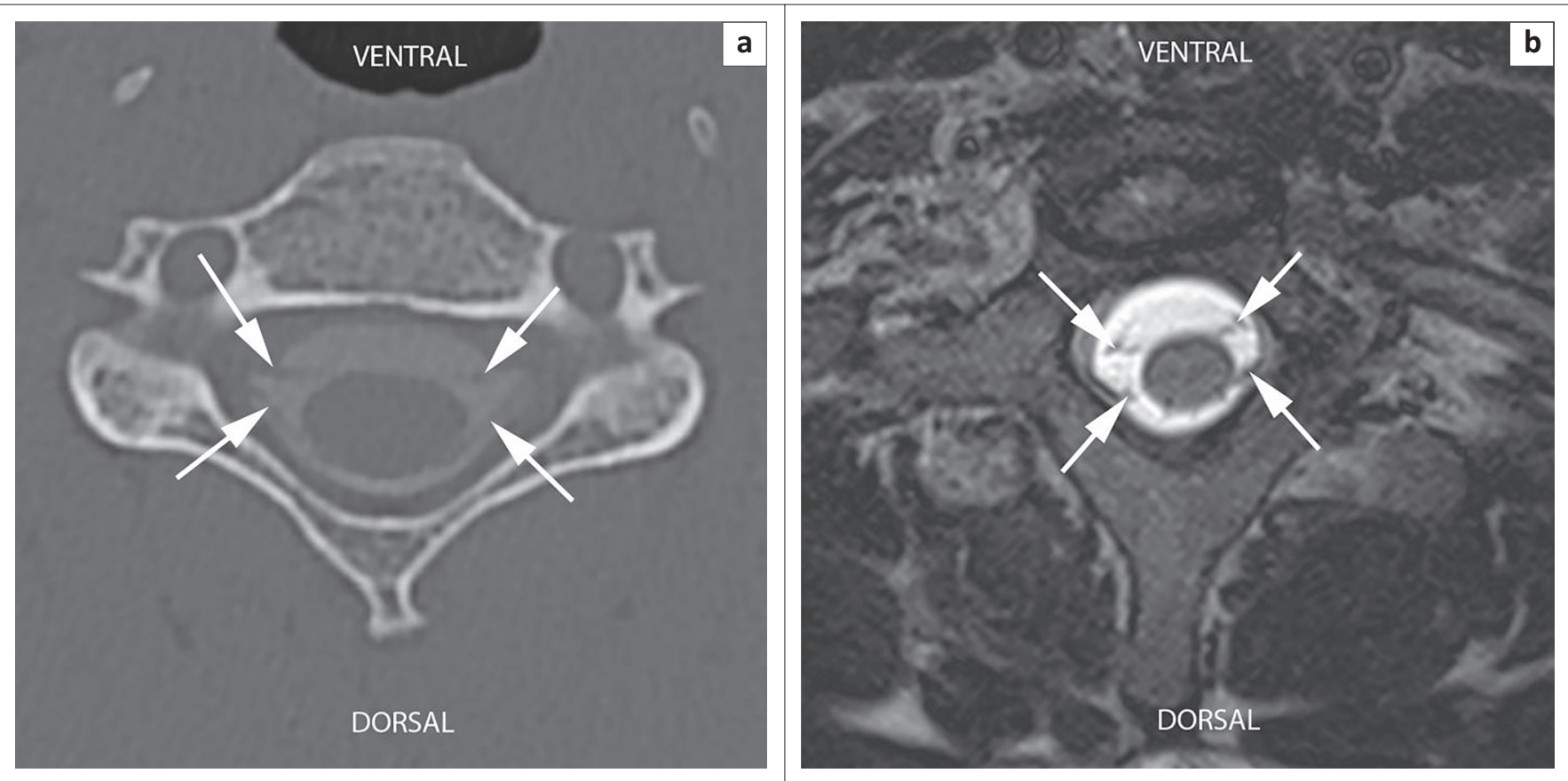

FIGURE 6: (a) Computed tomography myelography and (b) T2-weighted MRI axial images demonstrating normal ventral and dorsal preganglionic nerve roots (arrows) of the brachial plexus.
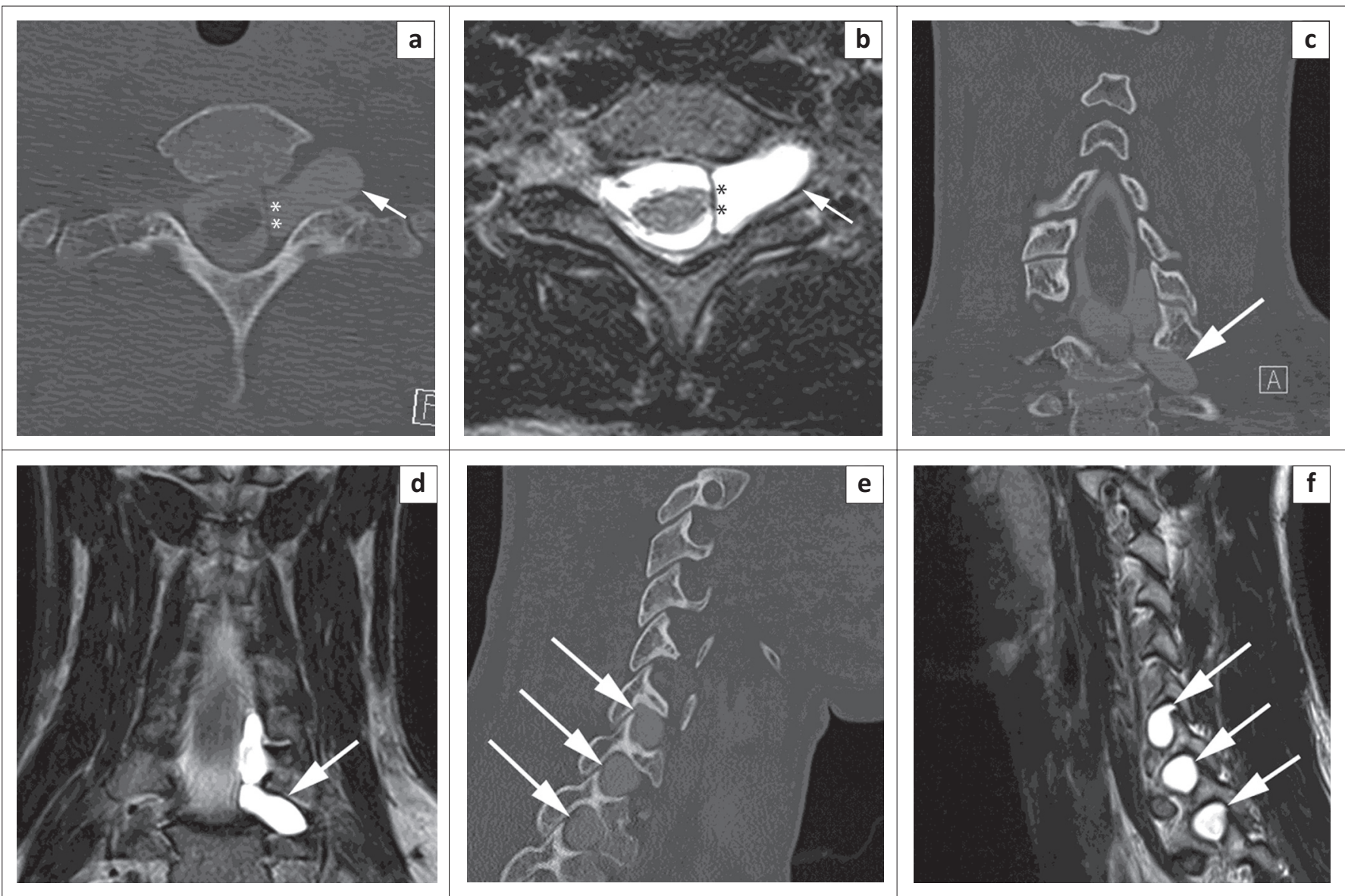

FIGURE 7: Pseudomeningoceles (arrows) detected within the same patient on computed tomography myelography (CTM) axial (a), coronal (c), sagittal (e) and $\mathrm{T}_{2}$-weighted magnetic resonance imaging (MRI) axial (b), coronal (d), sagittal (f) images. Axial images also demonstrate complete preganglionic nerve root avulsions (asterisk) of the left $\mathrm{C7}$ nerve roots (dorsal and ventral) detected on CTM (a) and $\mathrm{T}_{2}$-weighted MRI (b). 
TABLE 2: Advantages and disadvantages of MRI.

\section{Advantages of MRI over CTM}

Non-invasive procedure without intrathecal injection of contrast medium

High contrast resolution between nerve roots and perimedullary subarachnoid spaces

Excellent visualisation of the spinal cord

Permits visualisation of the distal brachial plexus

\section{Disadvantages of MRI}

It is difficult to use with claustrophobic patients

Its use is prohibited by ferromagnetic substances

Prominent cerebrospinal fluid flow artefacts (especially in patients with spinal stenosis) Presence of movement artefacts in uncooperative patients ${ }^{6}$

CTM, computed tomography myelography; MRI, magnetic resonance imaging.
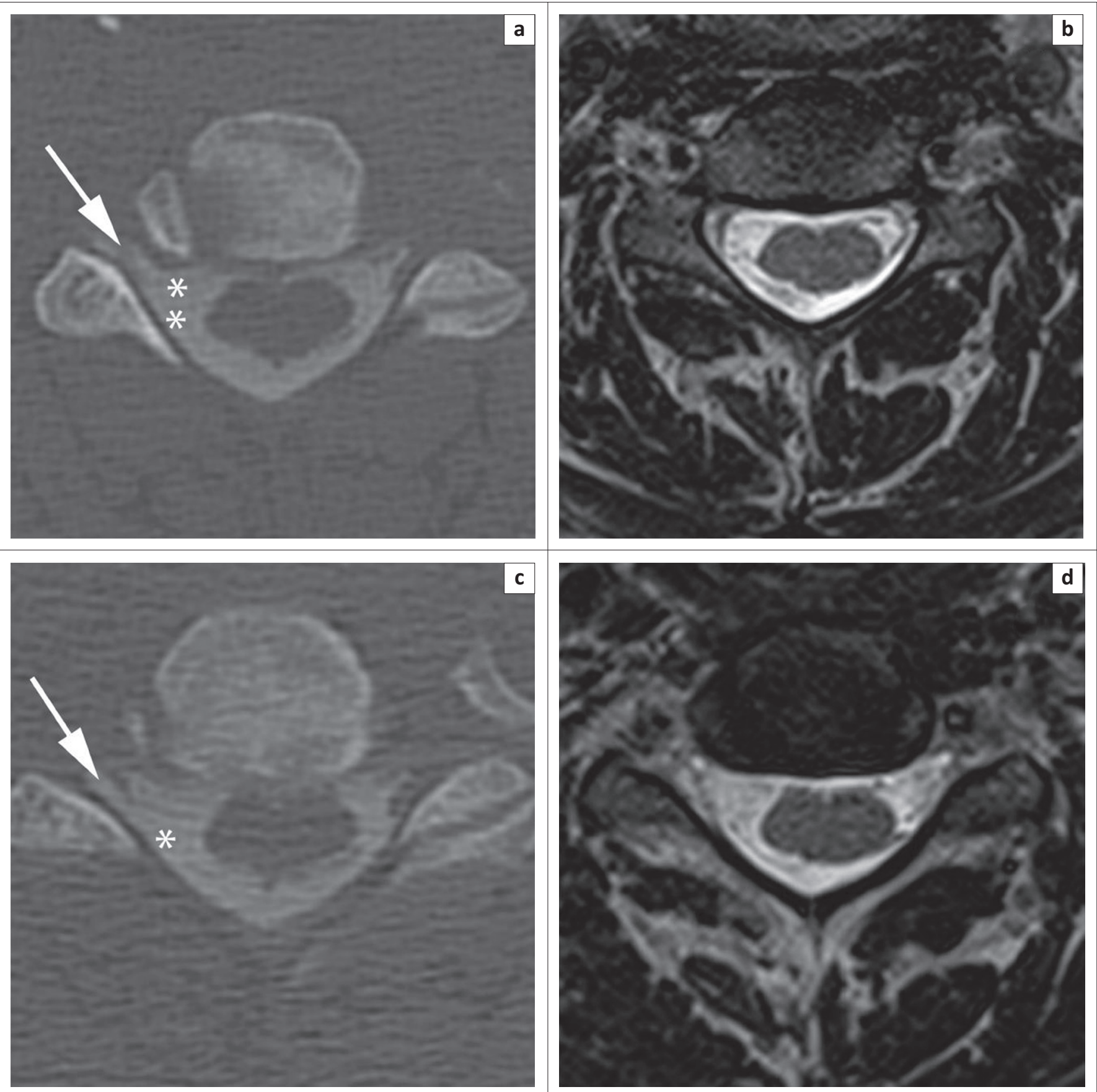

FIGURE 8: Discrepancies noted between CTM and MRI. Pseudomeningoceles (arrows) visualised on axial CTM images at the C5 (a) and C6 (c) nerve roots on the right, which was not visualised on axial $\mathrm{T}_{2}$-weighted magnetic resonance imaging (MRI) images (b, d) of the same nerve roots. Axial computed tomography myelography (CTM) images also demonstrated complete (dorsal and ventral) preganglionic nerve root avulsion (asterisk) at $\mathrm{C} 5$ on the right (a) with a partial preganglionic nerve root avulsion of the dorsal nerve root (asterisk) at C6 on the right (c). No preganglionic nerve root avulsion injuries could be visualised on axial T-weighted MRI of C5 (b) or C6 (d). 


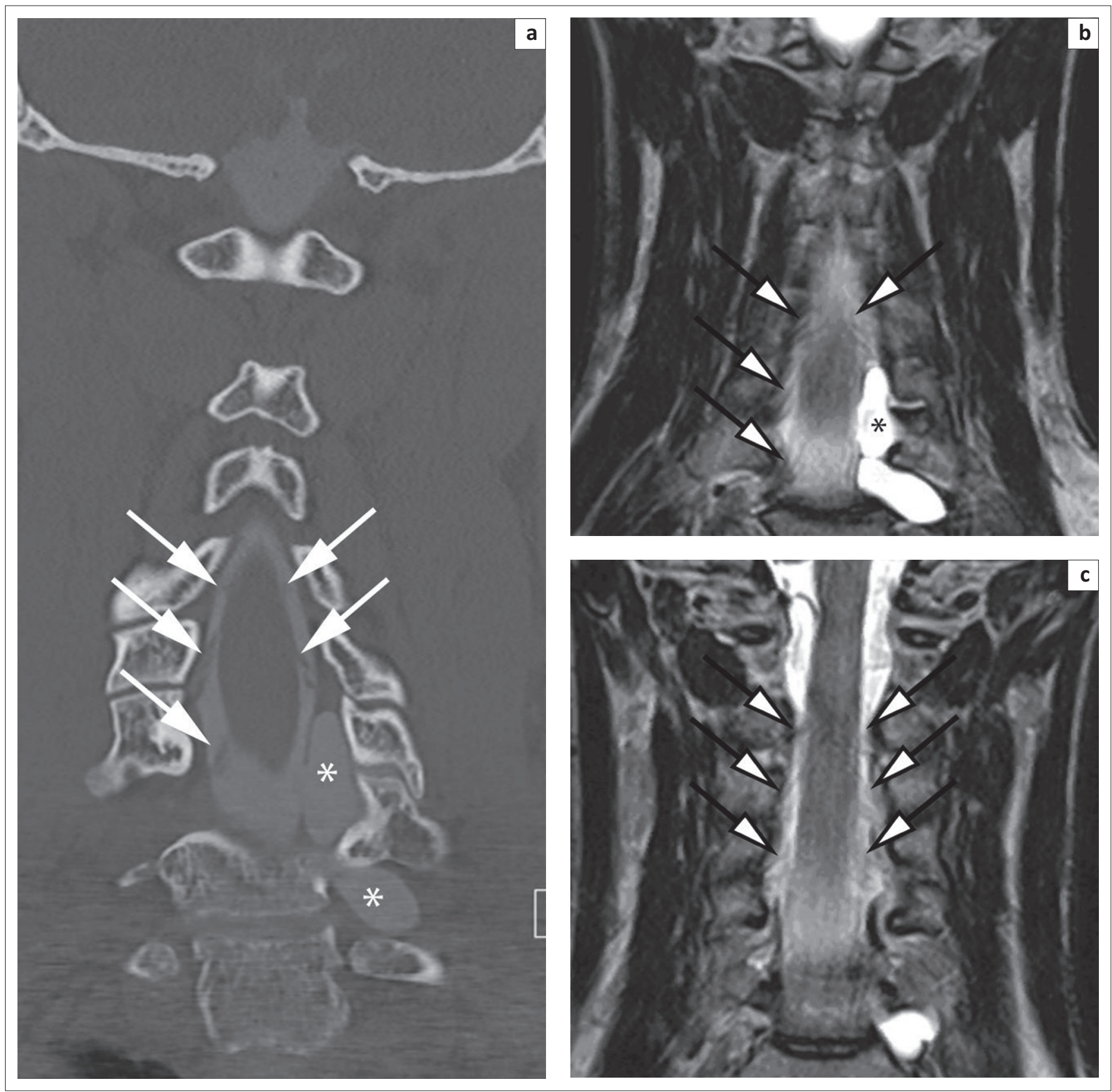

FIGURE 9: Coronal images demonstrating intact preganglionic nerve roots (arrows) on computed tomography myelography (CTM) (a) and $\mathrm{T}_{2}$-weighted magnetic resonance imaging (MRI) (b, c) together with left C7/C8 pseudomeningoceles and avulsed nerve roots (asterisk).

nerve roots run obliquely. Coronal and coronal-oblique views of CTM were therefore more advantageous for detecting nerve root avulsion injuries than axial views (Figure 9).

New MRI sequences are available, such as 3D CISS (threedimensional constructive interference in steady state). These enable the acquisition of thin slices to perform reconstruction in three different planes to increase diagnostic accuracy. Another new MRI technique is diffusion-weighted MR neurography (MRN); however, the main limitation of this technique is the lack of depiction of nerve roots above the level of C5. MRN can be done on $1.5 \mathrm{~T}$ or $3 \mathrm{~T}$, with the primary benefit of increased signal-to-noise ratio (SNR) of imaging at high field strength.
Diffusion-weighted imaging (DWI), diffusion tensor imaging (DTI) and DTI with tractography have also become valuable techniques in evaluating peripheral nerve pathologies. Further newer imaging sequences include T2 DRIVE, FIESTA and other heavily $\mathrm{T}_{2}$-weighted sequences. ${ }^{11,12}$

\section{Conclusion}

MRI was reported to be just as sensitive as CTM in detecting preganglionic nerve root avulsion injuries and pseudomeningoceles of spinal nerve roots C7-T1 of the brachial plexus. Some mild discrepancies existed at the $\mathrm{C} 5$ and C6 nerve root levels, with MRI detecting half of the injuries that were noted on CTM. 
Owing to the invasiveness of the procedure and associated patient discomfort, it is recommended that CTM be reserved for patients with contraindications to MRI or for complicated cases.

\section{Acknowledgements Competing interests}

The authors declare that they have no financial or personal relationships which may have inappropriately influenced them in writing this article.

\section{Authors' contributions}

E.V.D.L (University of KwaZulu-Natal) was the principal investigator and wrote the manuscript. V.N. (University of KwaZulu-Natal) was the supervisor of the study and together with A.M. (Inkosi Albert Luthuli Central Hospital) and A.R. (Inkosi Albert Luthuli Central Hospital) made conceptual contributions towards the study and participated in manuscript editing.

\section{References}

1. Moran SL, Steinmann SP, Shin AY. Adult brachial plexus injuries: Mechanism patterns of injury, and physical diagnosis. Hand Clin. 2005;21(1):13-24. http:// dx.doi.org/10.1016/j.hcl.2004.09.004
2. Yoshikawa T, Hayashi N, Yamamoto S, et al. Brachial plexus injury: Clinical manifestations, conventional imaging findings, and the latest imaging techniques. Radiographics. 2006;26:S133-S143. http://dx.doi.org/10.1148/rg.26si065511

3. Belzberg AJ, Storm PB, Moriarity JH. Surgical repair of brachial plexus injury: A multinational survey of experienced peripheral nerve surgeons. J Neurosurg. 2004;101:365-376. http://dx.doi.org/10.3171/jns.2004.101.3.0365

4. Carvalho GA, Nikkhah G, Matthies C, Penkert G, Samii M. Diagnosis of root avulsions in traumatic brachial plexus injuries: Value of computerized tomography and magnetic resonance imaging. J Neurosurg. 1997;86:69-76. http://dx.doi. org/10.3171/jns.1997.86.1.0069

5. Ozdoba C, Rieke A, Binggeli R, Schroth G. Myelography in the age of MRI: Why we do it, and how we do it. Radiol Res Pract. 2011; Art \#329017. http://dx.doi. org/10.1155/2011/329017

6. Gasparotti R, Garozzo D, Ferraresi S. Chapter 18 - Radiographic assessment of adult brachial plexus injuries. In: Chung KC, Yang LJS, McGillicuddy JE, editors. Practical management of pediatric and adult brachial plexus palsies. Philadelphia: W.B. Saunders, 2012; p. 234-248. http://dx.doi.org/10.1016/B978-1-4377-05751.00018-6

7. Altman DG. Practical statistics for medical research. London: Chapman and Hall; 1991.

8. Limthongthang R, Bachoura A, Songcharoen P, Osterman AL. Adult brachial plexus injury: Evaluation and management. Orthop Clin North Am. 2013;44(4):591-603. http://dx.doi.org/10.1016/j.ocl.2013.06.011

9. O'Shea K, Feinberg JH, Wolfe SW. Imaging and electrodiagnostic work-up of acute adult brachial plexus injuries. J Hand Surg Eur Vol. 2011;36(9):747-759. http:// dx.doi.org/10.1177/1753193411422313

10. Yamazaki H, Doi K, Hattori Y, Sakamoto S. Computerized tomography myelography with coronal and oblique coronal view for diagnosis of nerve root avulsion in brachial plexus injury. J Brachial Plex Peripher Nerve Inj. 2007;2:16.

11. Abul-Kasim K, Backman C, Björkman A, Dahlin LB. Advanced radiological work-up as an adjunct to decision in early reconstructive surgery in brachial plexus injuries. J Brachial Plex Peripher Nerve Inj. 2010;5:14-20.

12. Mallouhi A, Prayer D, Kainberger F, Bodner G, Kasprian G. 3T MR tomography of the brachial plexus: Structural and microstructural evaluation. Eur J Radiol. 2012;81:2231-2245. http://dx.doi.org/10.1016/j.ejrad.2011.05.021 